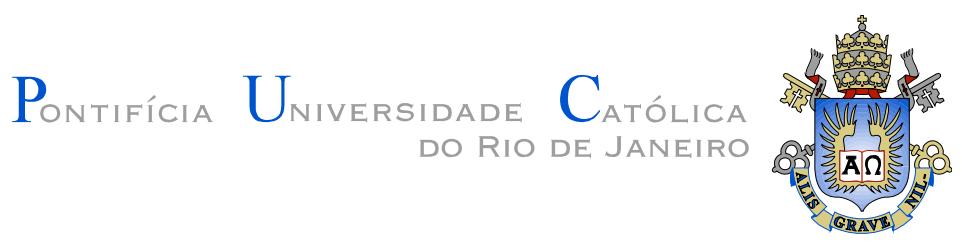

Carlos Eduardo Deodoro Rodrigues

\title{
Avaliação Metrológica da Resposta de Sinais de Fluorescência para Medições de Pigmentos em Água
}

Dissertação de Mestrado

Dissertação apresentada como requisito parcial para obtenção do título de Mestre pelo Programa de PósGraduação em Metrologia para Qualidade e Inovação da PUC-Rio.

Orientador: Prof. Dr. Raul Almeida Nunes Co-Orientador: Prof. Dr. Rui Pitanga Marques da Silva

Rio de Janeiro Setembro de 2007 

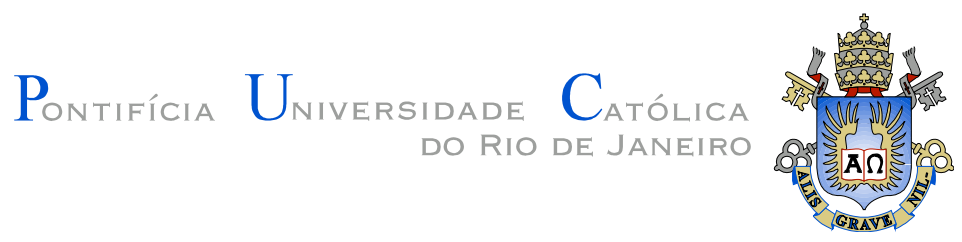

\section{Carlos Eduardo Deodoro Rodrigues}

\section{Avaliação metrológica da resposta de sinais de fluorescência para medições de pigmentos em água}

Dissertação apresentada como requisito parcial para obtenção do grau de Mestre pelo Programa de Pós-Graduação em Metrologia do Centro Técnico Científico da PUC-Rio. Aprovada pela Comissão Examinadora e homologada pela Coordenação Setorial de Pós-Graduação, formalizado pelas respectivas assinaturas.

Comissão Examinadora:

Prof. Dr. Raul Almeida Nunes Orientador Departamento de Ciência dos Materiais e Metalurgia (DCMM)

Pontifícia Universidade Católica do Rio de Janeiro

Prof. Dr. Rui Pitanga Marques da Silva Co-Orientador Programa de Pós- Graduação em Metrologia Pontifícia Universidade Católica do Rio de Janeiro

Dr. Paulo Cesar de Campos Barbosa PETROBRAS - Petróleo Brasileiro S.A.

Prof. Dr. Marcos Henrique de Pinho Maurício Departamento de Ciência dos Materiais e Metalurgia (DCMM)

Pontifícia Universidade Católica do Rio de Janeiro

Profa. Dra. Elisabeth Costa Monteiro

Programa de Pós- Graduação em Metrologia Pontifícia Universidade Católica do Rio de Janeiro

Coordenação Setorial de Pós-Graduação:

Prof. José Eugênio Leal

Coordenador Setorial de Pós-Graduação do

Centro Técnico Científico (PUC-Rio)

Rio de Janeiro, 11 de setembro de 2007. 
Todos os direitos reservados. É proibida a reprodução total ou parcial do trabalho sem autorização da universidade, do autor e do orientador.

\section{Carlos Eduardo Deodoro Rodrigues}

Graduou-se em Licenciatura em Física na UFRRJ (Universidade Federal Rural do Rio de Janeiro) em 2003. É professor de física da rede estadual de ensino desde 2005.

\section{Rodrigues, Carlos Eduardo Deodoro}

Avaliação metrológica da resposta de sinais de fluorescência pra medições de pigmentos em água / Carlos Eduardo Deodoro Rodrigues ; orientador: Raul Almeida Nunes; co-orientador: Rui Pitanga Marques da Silva. - 2007. 109 f. : il. ; $30 \mathrm{~cm}$

Dissertação (Mestrado em Metrologia)-Pontifícia Universidade Católica do Rio de Janeiro, Rio de Janeiro, 2007. Inclui bibliografia

1. Metrologia - Teses. 2. Monitoramento remoto. 3. Sistema LIDAR. 4. Fluorescência. 5. Pigmentos. 6. Clorofila. I. Nunes, Raul Almeida. II. Silva, Rui Pitanga Marques da. III. Pontifícia Universidade Católica do Rio de Janeiro. Programa de Pós-Graduação em Metrologia para Qualidade e Inovação. IV. Título. 


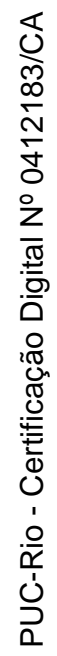

Dedico esta dissertação a minha família. 


\section{Agradecimentos}

À Deus, pela minha vida e por dela fazer parte cada uma das pessoas a quem agradeço aqui.

Aos meus pais, por terem sido meu suporte em todos os momentos da minha vida, pela educação que me deram, pelo amor com que sempre me cercaram e por terem me dado todo auxílio necessário para o sonho do mestrado tenha se tornado realidade.

À minha irmã Ana Paula, por ser parte da minha vida, por torcer por mim, por acreditar em mim e por ter comemorado cada uma das minhas conquistas até hoje como se fossem - e, de fato, também são - dela.

Aos meus amigos que estiveram sempre torcendo por mim e me incentivando das mais diferentes formas; todos de forma única; todos especiais e insubstituíveis em minha vida. Obrigado, Leandro, Roberta, Dri, Lu, Dani, Rafael, Zaira, Eduardo, Gustavo, Sgarbi, Diogo, Sérgio, Walace, Rodrigo e Érica.

Ao professor Raul por ser professor ao ensinar e amigo ao me aconselhar e apoiar, nos diversos momentos de idas e vindas neste período.

Ao professor Rui Pitanga por participar ativamente na reta final como se estivesse presente desde o início.

À Gloria pela força e por estar sempre pronta a ajudar.

Ao CNPq e à PUC-Rio, pelos auxílios concedidos, sem os quais este trabalho não poderia ter sido realizado.

Ao professor Maurício que conseguiu o impossível e tornou este trabalho possível. 


\section{Resumo}

Rodrigues, Carlos Eduardo Deodoro; Nunes, Raul Almeida; Silva, Rui Pitanga Marques da. Avaliação metrológica da resposta de sinais de fluorescência para medições de pigmentos em água. Rio de Janeiro, 2007. 109p. Dissertação de Mestrado - Programa de Pós-Graduação em Metrologia. Área de concentração: Metrologia para Qualidade e Inovação (Pós-MQI), Pontifícia Universidade Católica do Rio de Janeiro.

Objetivo. Fornecer confiabilidade às medições do LIDAR a partir: (i) do estabelecimento de padrões de configuração dos parâmetros do equipamento, na obtenção de sinais de fluorescência para medições de pigmentos em água; (ii) da avaliação de diferentes procedimentos para melhorar a relação sinal-ruído, preservando a integridade das bandas de interesse contidas no espectro de fluorescência; (iii) da verificação da repetitividade do equipamento para medições de comprimentos de onda associados aos máximos do espalhamento Raman da água, da banda de clorofila a, assim como, da banda de matéria orgânica dissolvida (MOD); (iv) e da avaliação da eficiência do processamento desenvolvido para obtenção de concentrações relativas da clorofila a quando comparadas aos resultados correlacionados à concentrações absolutas desse tipo de pigmento. Motivação. Contribuir para o desenvolvimento do monitoramento remoto utilizando um sistema LIDAR, fornecendo parâmetros que possibilitem maior confiabilidade às informações adquiridas pelo sistema. Contextualização. O monitoramento ambiental é uma prática adotada com maior freqüência por inúmeras empresas e instituições com o objetivo de minimizar impactos nocivos ao meio ambiente. O monitoramento remoto é uma ótima alternativa, pois, possibilita um levantamento prévio de possíveis vazamentos de diferentes tipos de poluição, sendo sensível à avaliação de sutis alterações no meio, possibilitando a identificação desses problemas antes mesmo de se tornarem aparentes. Metodologia. A prática adotada na obtenção de êxito dos objetivos 
apontados baseou-se: (i) na coleta de informação de diferentes modelos de monitoramento remoto, principalmente, utilizando um sistema LIDAR; (ii) na coleta de dados através da experimentação em laboratório e em campo e (iii) no desenvolvimento de processos que possibilitaram a análise das informações contidas nos sinais obtidos. Resultados. Na busca de um sinal-padrão de resposta do equipamento, o trabalho consiste em análises experimentais e no desenvolvimento de processos que auxiliem na análise das informações contidas nos sinais. Dentre os experimentos pode-se destacar duas abordagens: (i) a primeira, no laboratório, a partir de análise de amostras de água pura, que avalia as flutuações do sinal de fluorescência do espalhamento Raman da água para diferentes parâmetros do equipamento; (ii) e uma segunda análise, com dados in vivo, que avalia a concentração relativa de clorofila a para diferentes processos a fim de obter um processo padrão que diminui a sua incerteza na determinação desse quantitativo. Conclusões. O sensoriamento retomo é uma técnica útil para a investigação ambiental, porém, a implantação de práticas que contribuem para o aumento da confiabilidade dos dados fornecidos pelo sistema são igualmente indispensáveis.

\section{Palavras-chave}

Metrologia; Monitoramento Remoto; Sistema LIDAR; Fluorescência; Pigmentos; Clorofila. 


\section{Abstract}

Rodrigues, Carlos Eduardo Deodoro; Nunes, Raul Almeida; Silva, Rui Pitanga Marques da. Metrological Assessment of Fluorescence Signals of Measurements of Pigments in Water . Rio de Janeiro, 2007. 109p. Master dissertation. - Pos-graduation programme in Metrology. Concentration area: Metrology for quality and innovation. Pontifical Catholic University of Rio de Janeiro (PUC-Rio).

Aim. Providing reliability of LIDAR readings of fluorescence signals for measurements of pigments in ocean water by: (i) establishing configuration standards for equipment parameters. (ii) assessing different procedures to improve the signal-tonoise relationship. (iii) preserving the integrity of the wavelength bandwidth of the fluorescence spectrum. (iv) verifying the repeatability of wavelength measurements associated with local maxima of the Raman scattering curve of ocean water and chlorophyll a, as well as organic materials (dissolved in water) bandwidths. (v) and assessing the efficiency of the methodology developed for obtaining relative concentrations of chlorophyll a. Motivation. Contributing to the development of remote monitoring when using the LIDAR technique. This would bring about greater reliability. Context. Over the years environmental surveillance has been adopted by many companies and institutions as an effective practice to minimize harmful impacts on the environment. The remote monitoring is unquestionably a versatile alternative for detecting possible leaking of noxious substances before they become apparent. Methodology. The procedures included: (i) collecting information of different physical models of remote monitoring. (ii) collecting data from experiments carried out in the laboratory and in situ. (iii) and developing a procedure to analyze signal information. Results. Two approaches were adopted when carrying out the experiments: (i) in the laboratory, by evaluating fluorescence signal fluctuations of the Raman scattering in ocean water for different equipment parameters. (ii) and in situ, by measuring the relative concentration of chlorophyll a in ocean water for 
different schemes in order to choose the standard one. Conclusions. The remote monitoring is a useful tool to carry out environmental surveillance. But to increase the reliability of data collected by the optical-laser system apparatus the introduction of metrological assessment procedures is mandatory.

\section{Keywords}

Metrology; Remote Monitoring; LIDAR System; Fluorescence; Pigments; chlorophyll. 


\section{Sumário}

1 Introdução 19

2 Fundamentos Teóricos $\quad 28$

2.1. Processos de Decaimento Não-Radioativos 28

2.2. Fluorescência $\quad 30$

2.2.1. Concentração do Fluoróforo 32

2.3. Espalhamento Raman da Água 34

đิ 2.3.1. Variação da posição do máximo do espalhamento Raman com a temperatura 36

2.4. Fotossíntese 37

2.4.1. Fatores Limitantes da Fotossíntese 39

2.4.2. O Aparelho Fotossintético 40

2.4.3. Fotofosforilação 43

2.4.4. Ciclo de Calvin 46

2.5. Normalização do sinal de fluorescência pelo espalhamento Raman da água 47

3 Materiais e Métodos $\quad 49$

3.1. Descrição Geral do LIDAR 50

3.1.1. Sistema Laser $\quad 50$

3.1.1.1. Unidade Laser 51

3.1.1.2. Fonte de Alimentação 52

3.1.1.3. Q-Switch 53

3.1.1.4. Sistema de Refrigeração 53

3.1.2. Sistema Óptico 54

3.1.3. Sistema de Deteç̧ão 56

3.1.4. Sistema de Análise e Controle 58 
3.2. Calibração do Espectro $\quad 59$

3.3. Processamento do Sinal de Fluorescência de $\mathrm{Cl}$ a em Água 61

3.3.1. Correção da MOD pelo filtro QC13 62

3.3.2. Normalização do Espectro 63

3.3.3. Extração da Matéria Orgânica Dissolvida 64

3.3.4. Ajuste da Curva Gaussiana para o Espalhamento Raman da Água 65

3.3.5. Concentração Relativa de Clorofila 66

3.4. Montagem do equipamento $\quad 67$

3.4.1. Medições em laboratório $\quad 67$

3.4.2. Medições de campo 68

4 Experimentos e Resultados $\quad 70$

4.1. Estudo dos espectros de fluorescência obtidos pelo LIDAR 70

4.1.1. Avaliação da integridade das informações do sinal com a redução da base de dados do espectro $\quad 71$

4.1.2. Espectro médio para diferentes números de pulsos 74

4.1.3. Dispersão do sinal em cada detector 75

4.1.4. Dispersão do sinal após a passagem de um filtro de média móvel 78

4.2. Concentração relativa de clorofila a 81

4.2.1. Comparação dos resultados com média móvel para determinação da concentração de clorofila a em amostras de água costeiras 81

4.2.2. Comparação dos resultados com média móvel para determinação da concentração de clorofila a em amostras de água oceânicas 84

4.2.3. Correlação entre as concentrações relativas de clorofila a 87

4.2.4. Avaliação da sensibilidade do processo em relação à banda de clorofila a

4.3. Estudo dos parâmetros associados à banda do espalhamento Raman da água

4.3.1. Correlação entre os máximos do espalhamento Raman da água e da gaussiana 
4.3.2. Variação da posição do máximo do espalhamento Raman com parâmetros do equipamento

4.3.3. Cálculo da incerteza de medição da posição do máximo da banda do espalhamento Raman

4.3.4. Estudo da variação da posição do espalhamento Raman devido à passagem dos filtros de média móvel

4.3.5. Estudo das distribuições da concentração de clorofila a, MOD e intensidade do Raman para medições em águas oceânicas

5 Discussão e Conclusões 


\section{Lista de figuras}

Figura 1 - Esquema de decaimento dos elétrons através do processo de relaxação vibracional.

Figura 2 - Esquema da emissão radioativa por fluorescência de uma molécula após a absorção de radiação e diferentes processos de decaimento não-radioativo.

Figura 3 - Espectro de excitação e o espectro de emissão

fluorescente correspondente a essa faixa de absorção.

Figura 4 - Esquema dos espalhamentos Rayleigh e Raman.

Figura 5 - Relação entre a posição do máximo da gaussiana

ajustada à banda do espalhamento Raman e a temperatura da água.

Figura 6: Reagentes e produtos da fotossíntese.

Figura 7: Estrutura da molécula de ATP.

Figura 8: Visão geral da fotossíntese.

Figura 9: Espectros de absorção das clorofilas e outros pigmentos acessórios comparados ao espectro da luz solar, na região do visível, que atinge a superfície da Terra.

Figura 10: Estruturas internas dos cloroplastos.

Figura 11: Reações da Fotossíntese - reação dependente de luz, onde a energia luminosa é convertida em energia química de moléculas como ATP e NADPH; reação independente de luz, onde a energia molecular é utilizada na produção de moléculas de açúcar.

Figura 12: Reações que originam as moléculas de ATP e NADPH.

Figura 13: Diagrama simplificado do transporte de elétrons acíclico (não cíclico) no cloroplasto. Há dois centros de reação: PSI e PSII. Os elétrons são extraídos da água pelo PSII, transferidos para o PSI através de uma cadeia de transferência de elétrons e finalmente para o NADP+.

Figura 14: Reação de síntese de ATP. 
Figura 15: Ciclo de Calvin - Benson $\quad 47$

Figura 16 - Diagrama do LIDAR. 50

Figura 17 - O esquema de um laser de estado sólido, que consiste, em uma lâmpada, um cristal e espelhos altamente refletores. 52

Figura 18 - Esquema do sistema de refrigeração. 53

Figura 19 - Esquema óptico do telescópio newtoniano. 54

Figura 20 - Esquema óptico do policromador (vista lateral interna):

2. Espelho de deflexão, 3. Lente (autocolimação), 4. Rede de difração,

3. 5. Janela de entrada do intensificador de imagem.

Figura 21 - Esquema óptico do policromador (vista superior interna):

1. Fenda de entrada, 2. Espelho de deflexão, 3. Lente (autocolimação),

4. Rede de difração, 5. Janela de entrada do intensificador de imagem.

Figura 22 - Eficiência quântica dos detectores CCD.

Figura 23 - Diagrama de pulsos (1. mede o background antes de disparo do laser e 2. mede background mais o resultado da excitação da amostra).

Figura 24 - Parâmetros de Configuração (Linha superior: Controle da lâmpada; linha do meio: controle do Q-switch; linha inferior: controle do intensificador de imagens). Os valores embaixo de cada coluna especificam a duração de cada intervalo de tempo.

Figura 25 - Espectro de calibração - identifica o pixel correspondente aos comprimentos de onda $\lambda=472 \mathrm{~nm}$ e $\lambda=630 \mathrm{~nm}$.

Figura 26 - Espectro de fluorescência do LIDAR águas oceânicas ( $a$ - espalhamento Raman da água; b - MOD; c - Clorofila a)

Figura 27 - Curva de transmissão do filtro QC13.

Figura 28 - Matéria orgânica total corrigida pelo filtro QC13.

Figura 29 - (a) Espectro original de fluorescência, (b) Espectro normalizado pelo máximo do espalhamento Raman da água.

Figura 30 - Curva gaussiana (vermelho) que descreve os parâmetros da banda referente ao espalhamento Raman da água. Em verde temos a banda referente à clorofila $a$. 
Figura 31 - Espectro após a extração da banda do espalhamento Raman da água - a área verde evidencia a banda referente à clorofila $a$. 66

Figura 32 - Esquema do LIDAR no laboratório.

Figura 33 - Esquema da instalação do LIDAR e da posição de incidência do laser na superfície do mar em relação a embarcação (Barbosa, 2003). 69 Figura 34 - Espectro característico do LIDAR para uma amostra de água; a banda de comprimento de onda do espalhamento Raman da água centrada em 650,9 nm possui uma largura média de $24 \mathrm{~nm}$.

Figura 35 - O espectro de maior resolução é composto pelas informações de 454 detectores, enquanto que o espectro de menor resolução é composto pelas informações de 227 detectores. 73

Figura 36 - Dispersão do sinal medido em todos os detectores.

Figura 37 - Espectros médios de 320 pulsos do laser obtidos em intervalos de 4, 8, 16 e 32 pulsos do laser. (a) espectro médio de 80 espectros obtidos em intervalos de 4 pulsos do laser; (b) espectro médio de 40 espectros obtidos em intervalos de 8 pulsos do laser; (c) espectro médio de 20 espectros obtidos em intervalos de 16 pulsos do laser; (d) espectro médio de 10 espectros obtidos em intervalos de 32 pulsos do laser;

Figura 38 - Coeficiente de variação das medidas de cada detector para as diferentes seqüências de medição.

Figura 39 - Coeficiente de variação pixel a pixel de 20 espectros de 16 pulsos comparados à 80 espectros de 4 pulsos agrupados quatro a quatro.

Figura 40 - Dispersão pixel a pixel de 80 espectros de 4 pulsos com diferentes filtros de média móvel.

Figura 41 - Dispersão pixel a pixel de 20 espectros de 16 pulsos com diferentes filtros de média móvel.

Figura 42 - Dispersão das medidas de cada detector em torno da média para espectros de 4 pulsos agrupados de quatro em quatro espectros. Em ambos os grupos foram mantidos o número total de 320 pulsos. 
Figura 43 - Concentrações relativas de clorofila a para diferentes diluições obtidas a partir do processo padrão adotado no laboratório.

Figura 44 - Concentrações relativas de clorofila a para diferentes diluições obtidas, a partir do processo desenvolvido neste trabalho.

(a) sem filtro de média móvel; (b) com filtro de média móvel de 7 pontos;

(c) com filtro de média móvel de 15 pontos; (d) com filtro de média móvel de 31 pontos;

Figura 45 - Espectros médios obtidos a partir de amostras de águas costeiras; (a) espectro com filtro de média móvel de 7 pontos;

(b) espectro com filtro de média móvel de 15 pontos; (c) espectro com

(c) filtro de média móvel de 31 pontos;

Figura 46 - Espectros obtidos a partir de medições de amostras de água do mar; (A) espectro com filtro de média móvel de 7 pontos; (B) espectro com filtro de média móvel de 15 pontos;

Figura 47 - Distribuição das medidas de concentração da clorofila a para um conjunto de espectros obtidos de amostras de água oceânicas e filtrados com filtros média móvel de 7 e 15 pontos.

Figura 48 - Correlação entre os processos de extração da concentração de clorofila a com e sem filtros de média móvel. (a) média móvel de 7 pontos; (b) média móvel de 15 pontos; (c) média móvel de 31 pontos.

Figura 49 - Correlação entre o máximo do espalhamento Raman da água e o máximo da gaussiana ajustada a esta banda.

Figura 50 - A distribuição das medidas de posição do máximo do espalhamento Raman para as três configurações descritas na tabela 2. Os pontos vermelhos representam o valor médio da posição do máximo do Raman em cada distribuição.

Figura 51 - Distribuição das 20 repetições da medição da posição do máximo da banda do espalhamento Raman da água para o intervalo de 16 pulsos, onde LI é o limite inferior, LS é o limite superior e MED é o valor médio. $\left(F_{A}=500 \mathrm{~V} ; \mathrm{I} . \mathrm{I} .=750 \mathrm{~V} ; \bar{\lambda}=650,92 \mathrm{~nm}\right)$

Figura 52 - Cada ponto da distribuição corresponde à média de 
quatro medições da posição do espalhamento Raman da água no intervalo de 4 pulsos. Os limites superior (LS) e inferior (LI) distam $\pm 2 \sigma$ da média (MED). ( $\left.F_{A}=500 \mathrm{~V} ; \mathrm{I} . \mathrm{I} .=750 \mathrm{~V} ; \bar{\lambda}=650,45 \mathrm{~nm}\right)$

Figura 53 - Distribuição das posições do máximo do espalhamento Raman da água. (a) filtro de média móvel de 7 pontos; (b) filtro de média móvel de 15 pontos;

Figura 54 - Distribuição da concentração relativa de clorofila a e da concentração relativa da MOD para todas as estações medidas pelo LIDAR. As estações em destaque apresentam certa anomalia em relação às demais.

Figura 55 - Distribuição da concentração relativa de clorofila a e da intensidade do espalhamento Raman da água para todas as estações medidas pelo LIDAR. As estações em destaque apresentam resultados diferentes das demais.

Figura 56 - Distribuição da concentração relativa de MOD e da intensidade do espalhamento Raman da água para todas as estações medidas pelo LIDAR. As estações em destaque apresentam resultados diferentes das demais. 


\section{Lista de tabelas}

Tabela 1 - Quadro com os principais fatores limitantes da fotossíntese e

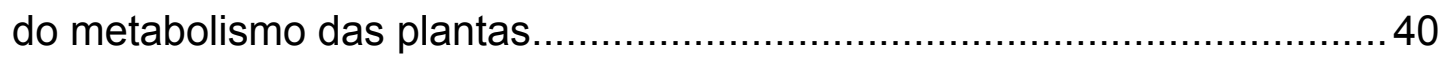

Tabela 2 - Características técnicas da unidade laser.................................. 51

Tabela 3 - Dados técnicos do sistema de refrigeração...................................54

Tabela 4 - $4 p$ - espectros adquiridos em intervalos de 4 pulsos do laser; $4 \times 4 p$ - agrupamento dos espectros de 4 pulsos quatro a quatro; $4 \times 4 p$ $\mathrm{c}$ / filtro - passagem de um filtro média móvel de 15 pontos no espectros de 4 pulsos agrupados quatro a quatro; $16 p$ - espectros adquiridos

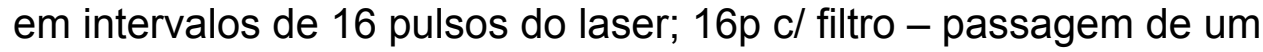
filtro média móvel de 15 pontos no espectros de16 pulsos; $32 p$ - espectros adquiridos em intervalos de 32 pulsos do laser.

Tabela 5 - Concentração relativa média de clorofila a para diferentes filtros de média móvel.

Tabela 6 - Coeficiente de correlação para as diferentes bandas de clorofila a.

Tabela 7 - Configurações da fonte de alimentação e do intensificador de imagem para cada 10 medições Tabela 8 - Resultados da medição da posição do espalhamento Raman da água. 